\title{
Nasal Probe Tests Decreased the Procedure Time During Flexible Bronchoscopy: A Randomized Controlled Trial
}

\section{Wenjun Zhu}

West China Hospital of Medicine: Sichuan University West China Hospital

\section{Yuchen Zhang}

West China Hospital of Medicine: Sichuan University West China Hospital

Jingyu Shi

West China Hospital of Medicine: Sichuan University West China Hospital

\section{Xiaoqin Wang}

West China Hospital of Medicine: Sichuan University West China Hospital

\section{Renjiao Li}

West China Hospital of Medicine: Sichuan University West China Hospital

Jia Liu

West China Hospital of Medicine: Sichuan University West China Hospital

\section{Dan Liu}

West China Hospital of Medicine: Sichuan University West China Hospital

Fengming Luo ( $\square$ fengmingluo@outlook.com )

Sichuan University West China Hospital https://orcid.org/0000-0001-9267-3437

\section{Research}

Keywords: Nasal probe test, flexible bronchoscopy, nasal cavity-glottis time, complications

Posted Date: November 13th, 2020

DOl: https://doi.org/10.21203/rs.3.rs-105235/v1

License: (c) (1) This work is licensed under a Creative Commons Attribution 4.0 International License. Read Full License 


\section{Abstract}

Background: Nasal insertion is the preferred method in flexible bronchoscopy; however, the relatively narrow nasal cavity increases the difficulty of bronchoscope insertion. The aim of the study was to investigate the advantages of a prior nasal cavity probe during flexible bronchoscopy and to investigate whether this novel method could reduce the complications associated with flexible bronchoscopy.

Methods: This prospective randomized controlled trial was conducted in a tertiary hospital between May 2020 and October 2020. Three hundred patients requiring diagnosis and treatment using flexible bronchoscopy were randomly allocated to three groups: a control group, a simple cotton bud detection group ( $C D$ group), and an adrenaline detection group ( $A D$ group). The outcomes were the time to pass the glottis, the first-pass intubation success rate, and postoperative complications, especially nasal bleeding.

Results: In total, 189 men and 111 women were enrolled in the study, with a mean age of $55.69 \pm 12.86$ years. The operation time was longer in the control group (24 [14.25-45.75] s) than in the CD group (19.5 [12.25-32.75] s). Compared with that in the CD group, the first-pass intubation success rate was significantly lower in the control group $(p<0.05)$. There was no difference in nasal bleeding between the control group and the CD group ( $p=0.506)$. Nasal bleeding after flexible bronchoscopy was more common in the $C D$ group than in the $A D$ group $(p=0.005)$; however, there was no significant between-group difference in hemostatic use $(p=0.245)$.

Conclusions: Use of a prior nasal cavity probe during flexible bronchoscopy can significantly reduce the time to pass the nasal cavity, improve the first-pass intubation success rate, and reduce postoperative nasal bleeding. Nasal detection is recommended as a time-saving procedure for patients undergoing flexible bronchoscopy.

Trial registration: ChiCTR2000032668, $6^{\text {th }}$ May.2020.

\section{Background}

Flexible bronchoscopy is a crucial method used in the diagnosis and treatment of pulmonary diseases that is widely used in hospitals, especially in the respiratory department. ${ }^{1}$ Nasal and oral insertion are the two most common approaches in clinical practice, with nasal insertion being preferred due to its advantages with respect to its simplicity, time-saving nature, and fewer complications. ${ }^{2}$ Topical lidocaine administered through the bronchoscope is better-tolerated with fewer complications, which contributes to the widespread use of topical anesthesia during flexible bronchoscopy. ${ }^{3-5}$ Although there have been several measures for improving patient comfort during the flexible bronchoscopy procedure, the relatively narrow nasal cavity and fragile nasal mucosa impede insertion, and a quick and blind removal may be necessary. ${ }^{6}$ The septum is not always located in the middle of the nasal cavity; moreover, the nasal passages on both sides are not of the exact same size. ${ }^{7}$ Therefore, the aim of the study was to 
investigate the superiority of prior probing of the nasal cavity during flexible bronchoscopy and to examine if this novel method could reduce the complications associated with flexible bronchoscopy.

\section{Methods}

This prospective randomized controlled trial was conducted in the Department of Pulmonary and Critical Care Medicine, West China Hospital of Sichuan University between May 2020 and October 2020 (chictr.org.cn,Identifier:ChiCTR2000032668). We enrolled inpatients and outpatients who required diagnosis and treatment using flexible bronchoscopy. The inclusion criteria were (1) diagnosis by a respiratory specialist with flexible bronchoscopy indications and (2) undergoing flexible bronchoscopy for the first time. The exclusion criteria were (1) aged < 18 years or > 85 years; (2) having decompensated heart failure, severe respiratory failure, history of upper airway surgery or radiation, a bleeding disorder, or mental illness; (3) requiring general anesthesia; and (4) having nasal disease requiring the orotracheal approach. This study was approved by the ethics committee of West China Hospital (Ethical approval No. 1044) and all the patients provided informed consent for voluntary study participation.

At enrollment, we collected the main demographics and clinical data of all patients. As per the protocol, all patients were required to fast for 4-6 hours before examination. Further, intravenous lines were established after entering the endoscopy room. Vital signs, including blood pressure, heart rate, and pulse oxygen saturation, were continuously monitored by the anesthesiologist and nurse during the flexible bronchoscopy. All patients received topical anesthesia using $2 \%$ lidocaine. Subsequently, the patients were allocated to the different study groups according to a computer-generated random number. In the control group, the bronchoscopy specialist chose the approach for flexible bronchoscopy according to experience and routine observation. In the simple cotton bud detection group (CD group), cotton buds with saline were used to probe the nasal cavity for a better approach. The nasal cavities on both sides were probed successively where the cotton bud was first inserted into the nasal cavity on the side with the least resistance. In the adrenaline detection group (AD group), cotton buds with a mixture of $1 \%$ oo adrenaline and $2 \%$ lidocaine were used to probe the nasal cavity for a better approach. All patients were unaware of the group allocation.

A bronchoscopy specialist used a BF-1TQ290 electronic bronchoscope (Olympus Optical Co., Tokyo, Japan) with an outer diameter of $6 \mathrm{~mm}$ to perform all the bronchoscopy procedures. Figure 1 shows the detailed procedural protocols and the relevant guidelines have been described previously ${ }^{8}$.

At the end of the flexible bronchoscopy procedure, the operation time (from touching of the nasal ostium to the touching of the glottis) was recorded. Moreover, the number of operations required for a successful procedure was recorded. In case the initial attempt for operators to reach the glottis through one nasal cavity side succeeded, the number of operations was recorded as one. If it failed on one side and succeeded on the other, the number of operations was recorded as two. Moreover, postoperative complications, including nasal bleeding and hemostatic use, were ascertained upon completion of the flexible bronchoscopy. 
Upon bronchoscopy completion, each patient was encouraged to fill a visual analog scale (VAS) to provide a rough estimate of pain, nasal foreign body sensation, and degree of comfort, with a maximum score of 100. Moreover, the operator filled out a 5-point VAS to assess the process of flexible bronchoscopy with 5 points indicating extremely successful.

\section{Statistical analysis}

All statistical analyses were performed using SPSS software (IBM SPSS, version 20, Chicago, IL). Data were presented as mean \pm SD or as a count (percentage). Intergroup comparison was performed using the independent-sample $t$ test, analysis of variance, Mann-Whitney $U$ test, or Fisher's exact test. Statistical significance was set at $p<0.05$.

\section{Results}

\section{Patients}

Between May 2020 and October 2020, 300 patients were randomly divided into three groups (each $n=$ 100) for routine flexible bronchoscopy examination and treatment. We enrolled 189 men and 111 women with a mean age of $55.69 \pm 12.86$ years. There were no significant between-group differences in age, sex, and body mass index ( $p>0.05$, Table 1$)$. The distribution of the disease types in the 3 groups was approximately balanced and did not affect the observation of outcomes. 
Table 1

Patient characteristics

\begin{tabular}{|c|c|c|c|c|c|}
\hline Variables & Control group & CD group & $A D$ group & Values & p-value \\
\hline & $(n=100)$ & $(n=100)$ & $(n=100)$ & & \\
\hline Age(years) & $53.33 \pm 14.21$ & $56.54 \pm 11.29$ & $57.30 \pm 12.70$ & $2.71^{\mathrm{a}}$ & 0.068 \\
\hline Sex(male/female) & $35 / 65$ & $35 / 65$ & $41 / 59$ & $1.03^{\mathrm{b}}$ & 0.598 \\
\hline $\begin{array}{l}\text { Body Mass Index } \\
(\mathrm{kg} / \mathrm{m} 2)\end{array}$ & $22.51 \pm 3.26$ & $22.39 \pm 2.93$ & $22.57 \pm 2.99$ & $0.09^{a}$ & 0.915 \\
\hline Indication & & & & $21.08^{b}$ & $0.275^{\dagger}$ \\
\hline COPD & 0 & 2 & 0 & & \\
\hline Space-occupying lesion & 55 & 65 & 58 & & \\
\hline Lung collapse & 4 & 2 & 1 & & \\
\hline Pneumonia & 27 & 19 & 31 & & \\
\hline Lung transplantation & 0 & 1 & 0 & & \\
\hline ILD & 1 & 2 & 1 & & \\
\hline Hemoptysis & 7 & 2 & 1 & & \\
\hline Pleural effusion & 1 & 2 & 4 & & \\
\hline Disease of the trachea & 4 & 4 & 3 & & \\
\hline Others & 1 & 1 & 1 & & \\
\hline \multicolumn{6}{|c|}{${ }^{a} \mathrm{~F}$ Values, ${ }^{\mathrm{b}} \mathrm{X} 2$ values, ${ }^{\dagger}$ Fisher's exact test used, Others = mediastinum space occupying lesion } \\
\hline COPD = chronic obstructi & pulmonary dis & Ise, ILD = Inters & tial lung diseas & & \\
\hline
\end{tabular}

During flexible bronchoscopy, the bronchoscopy specialist assessed the time taken for the bronchoscope to reach the glottis. Compared with the control group (24 [14.25-45.75] s), the CD group had a significantly shorter operation time (19.5 [12.25-32.75] s). The time data were converted by log (CD group: $1.31 \pm 0.30$ vs. control group: $1.40 \pm 0.33, p=0.039$ ) (Fig. 2a). Compared with that in the CD group, the number of operations until a successful procedure was significantly higher in the control group $(p=$ 0.015) (Fig. 2b). 
Figure 2a presents the nasal cavity-glottis time in both groups. The data are shown as mean \pm standard deviation. Figure $2 b$ presents the number of operations required for success in the two groups. Betweengroup comparisons were performed using the Mann-Whitney $U$ test.

\section{Postoperative complications}

Table 2 summarizes the occurrence of bleeding and hemostatic use after flexible bronchoscopy procedures. The number of patients who presented with nasal bleedings and required hemostatic use were $13(13 \%)$ and $5(38.46 \%)$ patients, respectively, in the control group; $10(10 \%)$ and $3(3 \%)$ patients in the $C D$ group; and $1(1 \%)$ and $0(0 \%)$ patients in the $A D$ group. There was no significant difference between the CD group and the control group for nasal bleeding $(p=0.506)$; however, nasal bleeding after flexible bronchoscopy was more common in the CD group than in the AD group $(p=0.005)$. Regarding hemostatic use, there was no significant difference between the CD group and the AD group $(p=0.245)$ (Table 2).

Table 2

Bleeding and use of hemostatic after flexible bronchoscopy procedures

\begin{tabular}{|lllll|}
\hline Variables & $\begin{array}{c}\text { CD group } \\
(\mathbf{n = 1 0 0 )}\end{array}$ & $\begin{array}{l}\text { AD group } \\
(\mathbf{n}=100)\end{array}$ & $\mathrm{X}^{2}$ values & P-value \\
\hline Bleeding after FB & & & 7.792 & 0.005 \\
\hline None & $90(90 \%)$ & $99(99 \%)$ & & \\
\hline Bleeding & $10(10 \%)$ & $1(1 \%)$ & & \\
Use of hemostatic & & & 1.354 & 0.245 \\
\hline None & $97(97 \%)$ & $100(100 \%)$ & & \\
\hline Use & $3(3 \%)$ & $0(0 \%)$ & & \\
\hline FB: flexible bronchoscopy & & & \\
\hline
\end{tabular}

\section{Tolerance and smoothness scores}

There were no between-group differences in the VAS scores for pain, foreign body sensation, degree of comfort toward flexible bronchoscopy, and the smoothness of the operation (all $p>0.05$, Table 3 ). 
Table 3

Tolerance and Smoothness Scores

\begin{tabular}{|llll|}
\hline Variables & Control group & Nasal probe group & P-value \\
\hline Pain & $(\mathrm{n}=100)$ & $(\mathrm{n}=200)$ & \\
\hline Foreign body sensation & $20(0-50)$ & $15(0-40)$ & $0.200^{\ddagger}$ \\
\hline Comfort degree & $40(20-60)$ & $30(20-50)$ & $0.134^{\ddagger}$ \\
\hline smoothness & $40(20-50)$ & $30(20-50)$ & $0.189^{\ddagger}$ \\
\hline $\begin{array}{l}\text { Visual analogue scale }(V A S) \text { scores for pain, foreign body sensation and comfort degree }(0=\text { non- } \\
\text { existent; } 100=\text { unbearable). VAS scores for the smoothness of the operation of the flexible } \\
\text { bronchoscopy }\left(5=\text { smoothest; } 0=\text { unbearable). }{ }^{\ddagger} \text { Mann-Whitney U test. }\right.\end{array}$ \\
\hline
\end{tabular}

\section{Discussion}

This prospective randomized controlled study investigated whether nasal probe tests could improve the flexible bronchoscopy procedure with respect to shortening the operation time and reducing postoperative complications. The results showed that probing the nasal cavity before flexible bronchoscopy significantly reduced the time to reach the glottis and improved the first-pass intubation success rate. Moreover, probing with cotton buds soaked in a mixture of $1 \%$ oo adrenaline and $2 \%$ lidocaine reduced nasal bleeding after flexible bronchoscopy.

Regarding the efficiency of this novel method, the results showed that flexible bronchoscopy with probing support reduced the manipulation time compared with the conventional approach. Probing with cotton buds allowed initial definition of the nasal cavity anatomy. Moreover, moist cotton buds could lubricate the nasal mucous membranes, which allows easier passage of the work channel through the nasal passages. Compared with the conventional approach, nasal detection resulted in time-saving for flexible bronchoscopy, which indicates that prior detection, rather than the bronchoscope itself, is more important for reducing time during the process. Moreover, the number of intubation attempts in the experimental group was lower than that in the control group, which confirmed the practical value of the timeconsuming flexible bronchoscopy.

Regarding the safety of this novel method, the results showed that it had fewer additional complications than the conventional approach. Bleeding is among the most concerning complications of bronchoscopy and endotracheal intubation. ${ }^{9.10}$ Apart from biopsy-related bleeding, bleeding caused by mechanical friction could cause anxiety and affect the quality of life of patients. We found that prior probing was associated with less nasal bleeding and that adrenaline, which reduced the insertion attempts, protected the nasal mucosa, shrunk the capillaries, and reduced bleeding. 
Since the introduction of the bronchoscope in 1966, it has become an increasingly important tool in the diagnosis and treatment of pulmonary diseases. ${ }^{11}$ Moreover, bronchoscopy has emerged as a tool for improving the management of complex lung diseases. ${ }^{12}$ Therefore, there is a need to maximize the utility of flexible bronchoscopy, simplify its operating process, and reduce postoperative complications. There have been several studies on bronchoscopy, ${ }^{13-16}$ however, there have been few studies on the examination of the nasal passages before bronchoscopy. To our knowledge, there has been no study on the influence of nasal cavity probing. Therefore, the current study shows that the use of nasal probe tests can reduce the postoperative complications which have clinical importance given the previously described side effects associated with flexible bronchoscopy. ${ }^{17}$ Second, nasal probe tests can decrease the procedure time and the number of operations performed before successful flexible bronchoscopy. They could enhance hospital single-day testing capabilities and reduce the incidence of bacteremia and post-bronchoscopy fever. ${ }^{18}$ Geertje et al described 66 flexible bronchoscopies in non-sedated adults. ${ }^{16}$ They found that the median time to reaching the glottis with nasal and oral insertion was $52.33 \mathrm{~s}$ and $24.43 \mathrm{~s}$, respectively, which was consistent with our findings. This shows that the time to pass the glottis could be reduced by smooth probing or bypassing the nasal cavity. Furthermore, the nasal probes are easy to perform, low cost, and easy to spread. Moreover, they can be applied in other medical procedures, including transnasal gastroscopy and nasotracheal intubation.

Regarding this study's strengths, this is the first prospective randomized controlled trial to assess the efficiency of probing the nasal cavity. The study sought to contribute to optimizing the nasal passage method during flexible bronchoscopy. This study has several limitations. First, nasal insertion was the first choice for the patients who underwent bronchoscopy; however, we used a 6.0-mm bronchoscope. Using a bronchoscope with a greater diameter could affect the time to pass the glottis and increase discomfort. There is a need for future studies using a 5.0-mm bronchoscope. Second, regarding bronchoscopy, the learning curve should be considered. Third, these findings have limited generalizability since this was a single-center study. Multicenter studies should confirm the reliability of these findings.

\section{Conclusions}

In conclusion, we recommend nasal probing as a time-saving procedure for patients undergoing flexible bronchoscopy that could shorten the time to reach the glottis and reduce the number of manipulation attempts.

\section{List Of Abbreviations}

Simple cotton bud detection group (CD group)

Adrenaline detection group (AD group)

Visual analog scale (VAS) 


\section{Declarations}

Ethics approval and consent to participate. This study was approved by the ethics committee of West China Hospital (Ethical approval No. 1044) and all the patients provided informed consent for voluntary study participation.

Consent for publication. Not applicable.

Availability of data and materials. The datasets analyzed during the current study are available from the corresponding author on reasonable request.

Competing interests. The authors declare that they have no competing interests.

Funding. This work was supported by grants from the National Nature Science Foundation of China (NSFC No.81770072), the "1.3.5 project for disciplines of excellence, West China Hospital, Sichuan University (ZYJC18021)", and Chengdu Science and Technology Bureau (No. 2018-CY02-00064-GX).

\section{Authors' contributions.}

Conceptualization: Dan Liu, Fengming Luo.

Formal analysis: Wenjun Zhu, Yuchen Zhang, Jingyu Shi.

Funding acquisition: Fengming Luo.

Investigation: Wenjun Zhu, Yuchen Zhang, Jingyu Shi, Xiaoqin Wang.

Methodology: Wenjun Zhu, Jingyu Shi, Xiaoqin Wang.

Project administration: Dan Liu, Fengming Luo.

Supervision: Dan Liu, Fengming Luo.

Visualization: Jia Liu.

Writing - original draft: Wenjun Zhu, Yuchen Zhang, Renjiao Li.

Writing - review \& editing: Renjiao Li, Fengming Luo.

Acknowledgments. The authors thank the patients who took part in the study and the staff of the Department of Pulmonary and Critical Care Medicine, West China Hospital, Sichuan University for their help with data collection.

\section{References}

1. Becker HD. Bronchoscopy: the past, the present, and the future. Clin Chest Med. 2010;31:1-18. 
2. Adewole OO, Onakpoya UU, Ogunrombi AB, Komolafe A, Odeyemi AD, Adeniran S, et al. Flexible fiberoptic bronchoscopy in respiratory care: diagnostic yield, complications, and challenges in a Nigerian Tertiary Center. Niger J Clin Pract. 2017;20:77-81.

3. Antoniades N, Worsnop C. Topical lidocaine through the bronchoscope reduces cough rate during bronchoscopy. Respirology, 2009;14:873-6.

4. Dreher M, Cornelissen CG, Reddemann MA, Müller A, Hübel C, Müller T. Nebulized versus standard local application of lidocaine during flexible bronchoscopy: a randomized controlled trial. Respiration. 2016;92:266-73.

5. Du Rand IA, Blaikley J, Booton R, Chaudhuri N, Gupta V, Khalid S, et al. British Thoracic Society guideline for diagnostic flexible bronchoscopy in adults: accredited by NICE. Thorax. 2013;68:i1-i44.

6. Song J. A comparison of the effects of epinephrine and xylometazoline in decreasing nasal bleeding during nasotracheal intubation. J Dent Anesth Pain Med. 2017;17.

7. Beeson WH. The nasal septum. Otolaryngol Clin North Am. 1987;20:743-67.

8. Wang F, Zheng $\mathrm{H}$, Zhang $\mathrm{Y}$, Zhu H, Shi J, Luo Y, et al. Nebulized Ipratropium bromide protects against tracheal and bronchial secretion during bronchoscopy: A randomized controlled trial. Medicine (Baltimore). 2019;98(47):e17942.

9. Terkawi RS, Altirkawi KA, Terkawi AS, Mukhtar G, Al-Shamrani A. Flexible bronchoscopy in children: Utility and complications. Int J Pediatr Adolesc Med. 2016;3:18-27.

10. Faiz, SA, Jimenez CA, Fellman BM, Huk T, Jazbeh S, Haque SA, et al. Incidence of bleeding complications with flexible bronchoscopy in cancer patients with thrombocytopenia. J Bronchology Interv Pulmonol. 2019;26:280-6.

11. Mahmoud N Fau - Kalanjeri, S. and S. Kalanjeri, Bronchoscopy BTI - StatPearls. 2020.

12. Miller RJ, Casal RF, Lazarus DR, Ost DE, Eapen GA. Flexible bronchoscopy. Clin Chest Med. 2018;39:1-16.

13. Barnes TW, Afessa B, Swanson KL, Lim KG. The clinical utility of flexible bronchoscopy in the evaluation of chronic cough. Chest. 2004;126:268-72.

14. Hsia D, DiBlasi RM, Richardson P, Crotwell D, Debley J, Carter E. The effects of flexible bronchoscopy on mechanical ventilation in a pediatric lung model. Chest. 2009;135:33-40.

15. Jain P, Sandur S, Meli Y, Arroliga AC, Stoller JK, Mehta AC. Role of flexible bronchoscopy in immunocompromised patients with lung infiltrates. Chest. 2004;125(2):712-

16. de Boer GM, Türk Y, Meuleman-van Waning VH, Braunstahl G. Bronchoscopy: Oral or nasal insertion? J Bronchology Interv Pulmonol. 2017;24:125-30.

17. Schramm D, Yu Y, Wiemers A, Vossen C, Snijders D, Krivec U, et al. Pediatric flexible and rigid bronchoscopy in European centers - availability and current practice. Pediatr Pulmonol. 2017;52:1502-8.

18. Kovaleva, J, Peters FTM, van der Mei HC, Degener JE. Transmission of infection by flexible gastrointestinal endoscopy and bronchoscopy. Clin Microbiol Rev. 2013;26:231-54. 
Figures

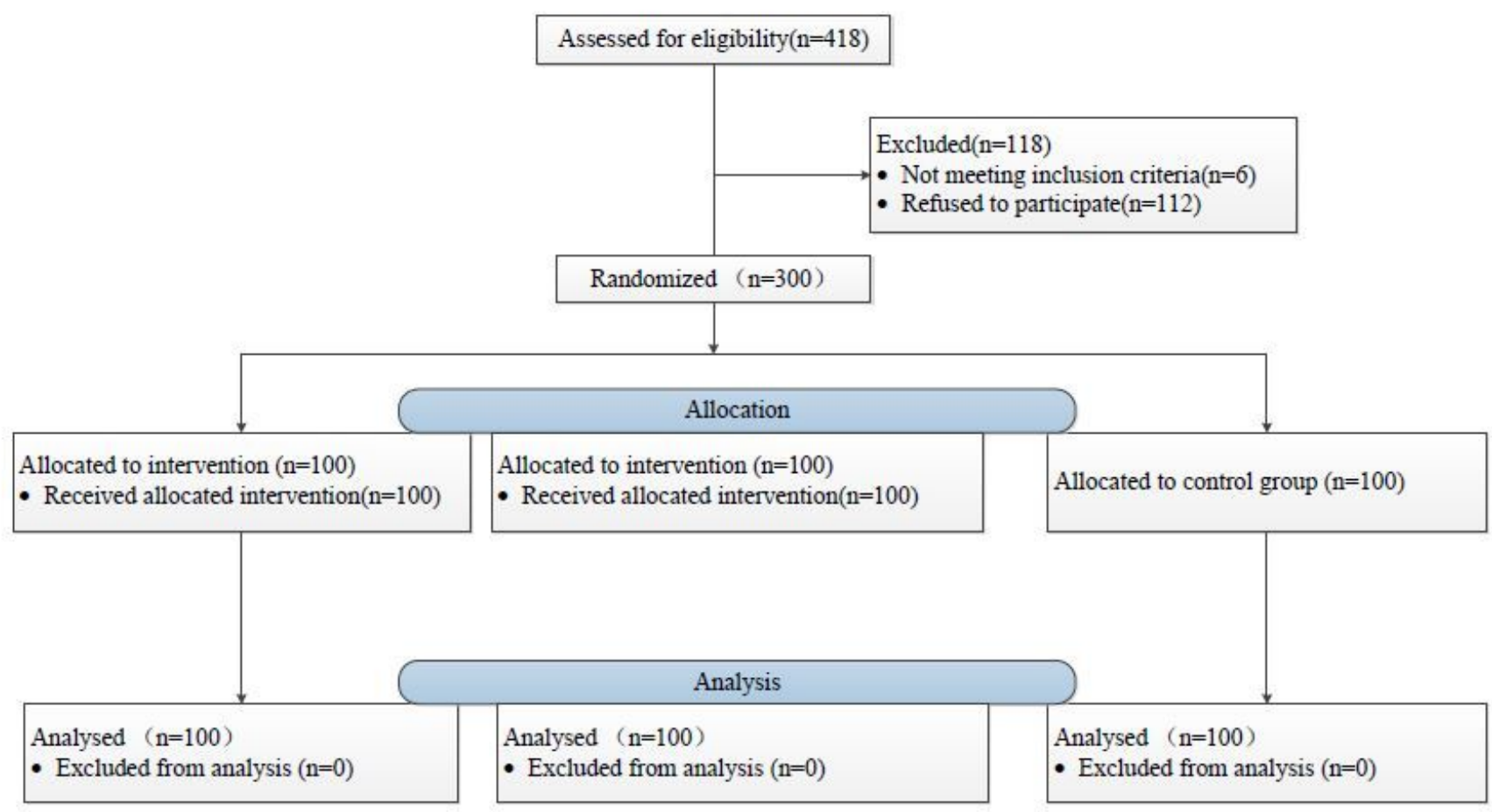

Figure 1

Flow chart illustrating patient selection throughout the trial. 


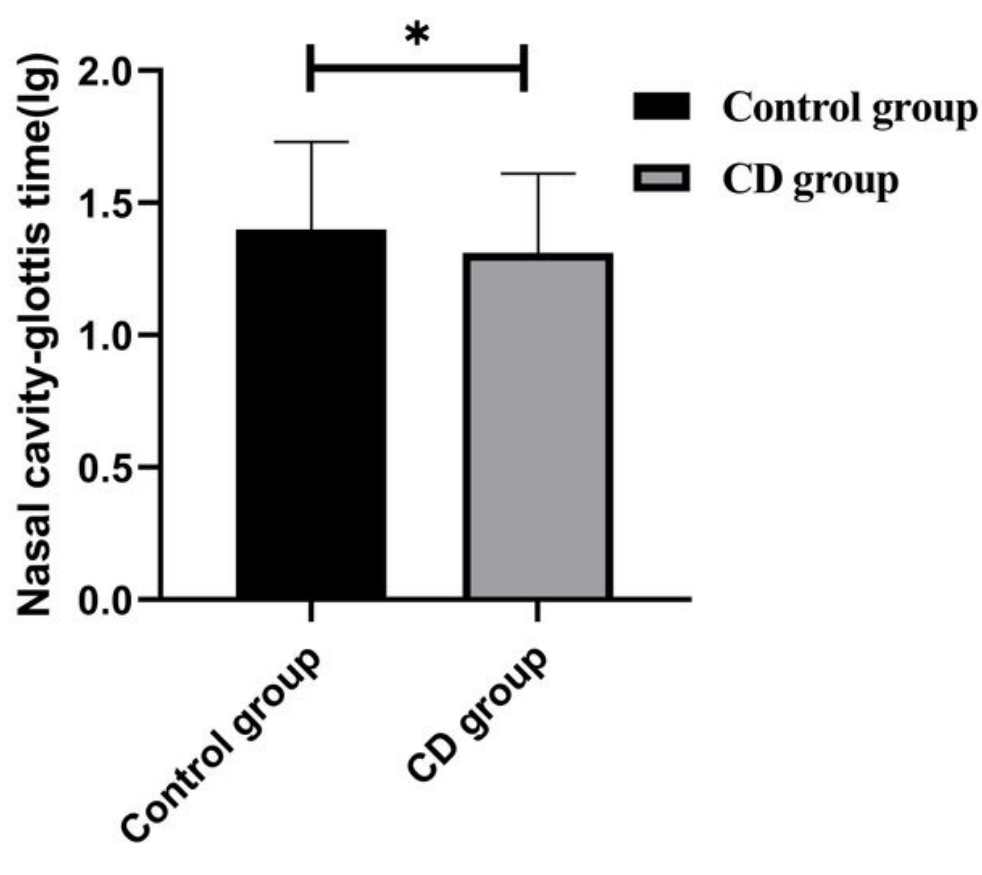

A

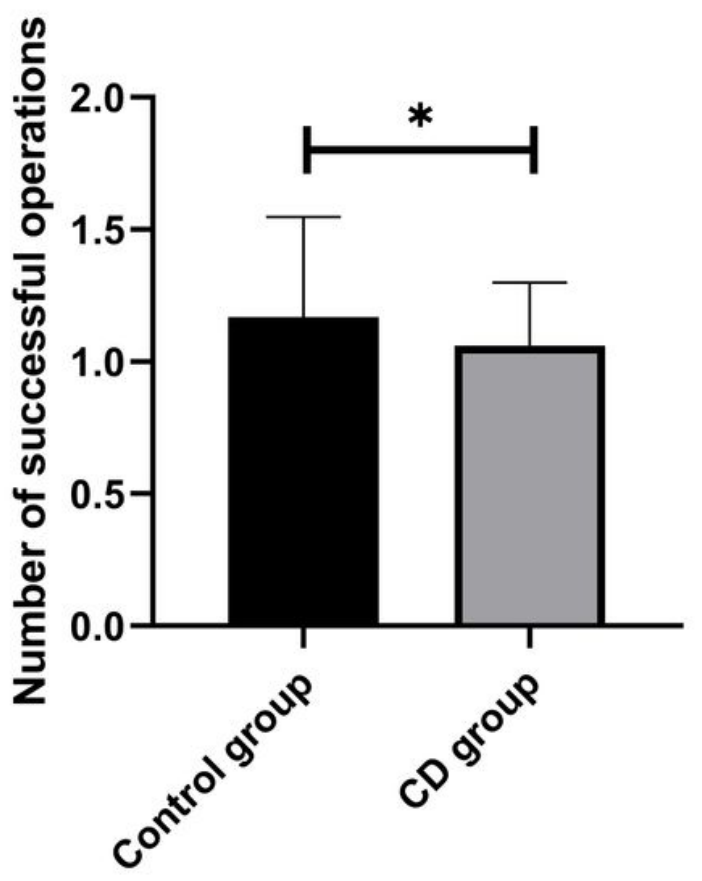

B

Figure 2

a. Nasal cavity-glottis time in two groups. Data are given as mean \pm standard deviation. $b$. The number of operations required for success in two groups. A two-group comparison was performed using the MannWhitney U test. 